\title{
Highly predictive SNP markers for efficient selection of the wheat leaf rust resistance gene $\operatorname{Lr} 16$
}

\author{
Mulualem T. Kassa ${ }^{1,2}$, Frank M. You', Colin W. Hiebert', Curtis J. Pozniak ${ }^{3}$, Pierre R. Fobert ${ }^{2}$, Andrew G. Sharpe ${ }^{2,4}$, \\ James G. Menzies ${ }^{1}$, D. Gavin Humphreys ${ }^{5}$, Nicole Rezac Harrison ${ }^{6}$, John P. Fellers ${ }^{7}$, Brent D. McCallum ${ }^{1}$ \\ and Curt A. McCartney ${ }^{1 *}$
}

\begin{abstract}
Background: $L r 16$ is a widely deployed leaf rust resistance gene in wheat (Triticum aestivum L.) that is highly effective against the North American Puccinia triticina population when pyramided with the gene Lr34. Lr16 is a seedling leaf rust resistance gene conditioning an incompatible interaction with a distinct necrotic ring surrounding the uredinium. Lr16 was previously mapped to the telomeric region of the short arm of wheat chromosome 2B. The goals of this study were to develop numerous single nucleotide polymorphism (SNP) markers for the Lr16 region and identify diagnostic gene-specific SNP marker assays for marker-assisted selection (MAS).

Results: Forty-three SNP markers were developed and mapped on chromosome 2BS tightly linked with the resistance gene Lr16 across four mapping populations representing a total of 1528 gametes. Kompetitive Allele Specific PCR (KASP) assays were designed for all identified SNPs. Resistance gene analogs (RGAs) linked with the Lr16 locus were identified and RGA-based SNP markers were developed. The diagnostic potential of the SNPS co-segregating with Lr16 was evaluated in a diverse set of 133 cultivars and breeding lines. Six SNP markers were consistent with the Lr16 phenotype and are accurately predictive of Lr16 for all wheat lines/cultivars in the panel.
\end{abstract}

Conclusions: Lr16 was mapped relative to SNP markers in four populations. Six SNP markers exhibited high quality clustering in the KASP assay and are suitable for MAS of Lr16 in wheat breeding programs.

Keywords: Lr16, Leaf rust, Puccinia triticina, Wheat, Triticum aestivum L, Disease resistance, Single nucleotide polymorphism (SNP), Linkage analysis, Resistance gene analog (RGA)

\section{Background}

Wheat (Triticum aestivum L.) is an important food crop providing a fifth of the world's calorie intake. Wheat is grown on more than 215 million hectares and cultivated across more regions of the world than any other staple crop [1]. Wheat diseases caused by various pathogens cause significant yield loss across the world. Of these, the rust fungi cause serious diseases of wheat and pose significant constraints to wheat production. The three wheat rust diseases are stem, leaf, and stripe (or yellow) rust, caused by Puccinia graminis f. sp. tritici Eriks. \& E.

\footnotetext{
* Correspondence: Curt.McCartney@agr.gc.ca

${ }^{1}$ Agriculture and Agri-Food Canada, Morden Research and Development

Centre, 101 Route 100, Morden, MB R6M 1Y5, Canada

Full list of author information is available at the end of the article
}

Henn., Puccinia triticina Eriks., and Puccinia striiformis Westend. f.sp. tritici Erikss., respectively [2].

Wheat leaf rust occurs more frequently and is more prevalent globally than other cereal rust pathogens $[3,4]$. In the eastern prairies of Canada, annual yield losses due to leaf rust are estimated at 5 to $15 \%$ when susceptible wheat cultivars are grown [4]. Leaf rust can be effectively controlled by applying fungicides or through genetic resistance. Of these, host resistance is the most efficient, economical, and environmentally effective means to control this disease. In addition, there is the possibility that rust pathogens may develop resistance to fungicides [5]. To date, 73 numerically designated wheat leaf rust resistance $(L r)$ genes have been identified and catalogued [6-8]. Additionally, a small number of race-specific seedling 
stage leaf rust resistance genes have been cloned, including $L r 1, L r 10$, and $L r 21$ [9-11]. All of these resistance (R) genes were found to belong to the coiled-coil, nucleotide binding site, leucine rich repeat (CC-NB-LRR) class of $\mathrm{R}$ proteins. $\operatorname{Lr} 34$ was the first wheat resistance gene to be cloned that provides partial resistance to multiple pathogens [12]. More recently, the partial leaf rust resistance gene $\operatorname{Lr67}$ was also cloned [13]. Lr34 and Lr67 encode a putative $\mathrm{ABC}$ transporter and a hexose transporter, respectively. Lr16 is likely a member of the CC-NB-LRR class of $\mathrm{R}$ proteins given its activity at the seedling stage and race-specificity. Although many wheat leaf rust $(L r)$ resistance genes have been identified, virulence mutations in the $P$. triticina population have overcome host resistance conferred by a number of $L r$ genes (i.e., race-specific $\mathrm{R}$ genes). To mitigate the problem posed by the evolution of new virulent races of the pathogen, deployment of multiple Lr genes or gene pyramiding is recommended to promote the durability of $\mathrm{Lr}$ genes [2].

Lr16 is a seedling leaf rust resistance gene, conditioning an incompatible interaction with a distinct necrotic ring surrounding the uredinium [14]. In Canada, Lr16 was first deployed in the variety Selkirk [4]. Virulence to Lr16 was first detected in Canada in 1961. Although considered a defeated gene, $\operatorname{Lr} 16$ still retains a partial resistance effect against virulent $P$. triticina isolates and still offers protection against wheat leaf rust in Canada $[14,15]$. It is widely deployed and is particularly effective against the North American P. triticina population when pyramided with $\operatorname{Lr} 34$ [16-18]. The exact nature of the interaction with $L r 34$ is not understood, but similar interactions were reported between $\operatorname{Lr} 34$ and $\operatorname{Lr} 1, \operatorname{Lr} 2 a$, Lr3, Lr3ka, Lr11, Lr13, Lr17, and LrB [16]. Genetic analysis has revealed the presence of $L r 16$ in the Canadian wheat cultivars 'AC Domain', 'AC Karma', 'AC Majestic,'AC Splendor,' 'Columbus', the American cultivar 'Grandin', and in other cultivars elsewhere [14, 19]. Resistance conferred by Lr16 has been utilized in wheat breeding programs in Canada and around the world.

Lr16 has been mapped to the terminal region of wheat chromosome arm 2BS [14, 20]. Previous studies have identified simple sequence repeat (SSR) markers that were linked with $\operatorname{Lr} 16$ on chromosome arm 2BS $[14,20]$. However, lack of distally flanking markers has hampered the fine mapping and cloning efforts of Lr16 and slowed the development of diagnostic molecular markers for marker-assisted selection (MAS). Thus, the main objectives of the present study were to map Lr16 in multiple mapping populations, develop numerous SNP markers for the Lr16 region, and identify SNP markers that are tightly linked with Lr16 and useful for efficient selection of Lr16 in wheat breeding programs.

\section{Methods}

\section{Mapping populations}

Four mapping populations were used in this study. An $\mathrm{F}_{6}$-derived recombinant inbred line (RIL) population ( $n$ $=384$ ) was developed from the cross of BW278 ('AC Domain" 2/'Sumai 3') with 'AC Foremost' (HY320*5/ BW553//HY320*6/7424-BW5B4; [21]). BW278 is a Canadian spring wheat breeding line that carries $\operatorname{Lr} 16$ (resistant parent) and 'AC Foremost' is Canadian spring wheat cultivar that is susceptible to $P$. triticina isolate 12-3 MBDS at the seedling stage. The 'AC Majestic'/ 'Glenlea' population consisted of $400 \mathrm{~F}_{1}$-derived doubled haploid (DH) lines. 'AC Majestic' ('Columbus'*2//'Saric 70 /'Neepawa'/3/'Columbus'"5//'Saric70'/'Neepawa') is a Canadian spring wheat cultivar that carries Lr16 (resistant parent) while 'Glenlea' (UM530/CB100; [22]) is a Canadian spring wheat cultivar that is susceptible to $P$. triticina isolate 12-3 MBDS at the seedling stage. The RL4452/'AC Domain' $F_{1}$-derived doubled haploid population $(n=172)$ was generated from the cross of the susceptible wheat line RL4452 ('Glenlea" $6 /{ }^{\prime} \mathrm{Kitt}$ '), which is susceptible to $P$. triticina isolate $12-3 \mathrm{MBDS}$ at the seedling stage, with the resistant (Lr16 carrier) Canadian spring wheat 'AC Domain' (ND499/RL4137//ND585; [23]). The fourth population was developed from a RIL population of 94 lines of the cross 'Kenyon'/86ISMN 2137. 'Kenyon' ('Neepawa'*5/'Buck Manantial'; [24]) is a Canada Western Red Spring (CWRS) cultivar that carries Lr16 (resistant), while 86ISMN 2137 is of unknown origin and is susceptible to $P$. triticina isolate 12-3 MBDS at the seedling stage. The 'Kenyon'/ 86ISMN 2137 mapping population was developed and provided by Dr. G.R. Hughes, University of Saskatchewan, Crop Development Centre. All other mapping populations were developed by the authors at Agriculture and Agri-Food Canada.

\section{Seedling tests with race MBDS (12-3)}

Tests for leaf rust resistance conditioned by Lr16 were done at the seedling stage as previously described [25]. Seeds were planted in clumps of approximately 10 seeds evenly spaced in fibre flats $(25 \times 15 \mathrm{~cm})$. Approximately $14 \mathrm{~d}$ after seeding, the seedlings at the two leaf stage were inoculated with urediniospores of $P$. triticina isolate 12-3 MBDS (nomenclature as previously described [26]) mixed with a light mineral oil (Bayol, Esso Canada, Toronto, ON) sprayed onto the leaves using a compressed air sprayer. This $P$. triticina isolate is fully avirulent on Lr16 carriers. The plants were allowed to dry, to evaporate the mineral oil, for at least $1 \mathrm{~h}$ then moved to a $100 \%$ humidity cabinet for approximately $17 \mathrm{~h}$ of incubation. The plants were then moved to a greenhouse at $20 \pm 4 \quad{ }^{\circ} \mathrm{C}$ with supplemental lighting. After approximately $14 \mathrm{~d}$, plants were rated for symptoms using a ' 0 ' 
to ' 4 ' infection scale where '0' (no symptoms), ';' (hypersensitive flecks), ' 1 ' (small uredinia with necrosis), and '2' (small to medium-sized uredinia with chlorosis) were considered resistant responses and ' 3 ' (medium-sized uredinia without necrosis or chlorosis) and '4' (largesized uredinia without necrosis or chlorosis) were considered susceptible responses [26].

\section{DNA marker development}

SSR markers linked to $\operatorname{Lr} 16$ were identified for analysis based on previous research [14]. Previous studies mapped Lr16 to the terminal region of wheat chromosome bin 2BS3 [fraction length: 0.84-1.00]. All sequences of previously mapped SSR, Diversity Arrays Technology (DArT), and SNP markers near Lr16 were used as queries in the BLASTN search of the Brachypodium genome to identify orthologous loci. Wheat EST (wEST) that were mapped to bin 2BS3 (FL 0.84-1.00) and publically available wEST sequences which are orthologous to putative Brachypodium genes within the syntenic genomic region on Chromosome 5 were used for developing conserved primers using ConservedPrimers 2.0 software [27].

SNP markers were developed with multiple strategies. Linked wheat $90 \mathrm{~K}$ Infinium SNP markers [28] were identified by genotyping the RL4452/'AC Domain' and BW278/'AC Foremost' mapping populations. KASP assays were designed for these SNPs and tested on the other populations.

In addition, a BLAST search using sequences of previously mapped markers on chromosome arm 2BS was performed to identify putative SNP markers linked to the $\operatorname{Lr} 16$ locus. The wheat $90 \mathrm{~K}$ iSelect Infinium assay [28] and the SNP database at CerealsDB [29] were used to mine SNPs. Additional SNP markers were also developed through comparative synteny analysis using Brachypodium and rice genomes. Here, syntenic Brachypodium and rice genes were used as queries in BLASTN searches against the wheat chromosome arm 2BS survey sequence to identify putative syntenic wheat genes. These wheat genes were then used as queries in BLASTN searches of the CerealsDB SNP database [29]. Genomic resources at NCBI [30] and GrainGenes [31] were utilized to discover additional SNP markers linked with $\operatorname{Lr} 16$.

Whole exome capture (WEC) data [32] of chromosome 2BS was also utilized to mine SNP markers associated with Lr16. Bulked segregant analysis (BSA) coupled with WEC sequencing was used to identify SNPs. Four sets of DNA (BW278 [Lr16-carrier], bulk of 15 Lr16-carrier BW278/'AC Foremost' RILs, 'AC Foremost' [non-carrier], and bulk of 15 susceptible BW278/'AC Foremost' RILs) were sequenced with Illumina short read technology $(2 \times 100 \mathrm{bp})$ and assembled against the WEC reference sequence. SNP variants were called that accurately differentiated the resistant and susceptible lines and bulks, respectively.

Finally, all potential genes from chromosome arm 2BS survey sequence of hexaploid wheat variety Chinese Spring [33] were predicted using gene prediction software GeneMark [34]. Resistance gene analogs (RGAs) from the genes on 2BS were identified using the RGA prediction pipeline program RGAugury [35], although Chinese Spring does not have Lr16. RGAs linked with the Lr16 locus were detected using previously mapped markers. BLAST was used to identify wheat RGAs colinear with RGAs in Brachypodium and rice as described above. RGA-based SNP markers were developed from sequences of RGAs Sanger sequenced in wheat lines with and without Lr16. Moreover, a BLAST search of SNPs from the CerealsDB wheat SNP database [29] and the $90 \mathrm{~K}$ wheat Infinium SNP array was conducted using sequences of the filtered RGA contigs as queries to identify SNP markers putatively linked to the Lr16 locus. RGA-based markers linked with Lr16 were also identified from RNA sequences (RNA-seq) and an in silico subtraction method [36]. Polymorphic SNPs were genotyped on the appropriate mapping populations and used for linkage analysis.

\section{DNA marker analyses and genotyping}

Genomic DNA for the populations, 'AC Majestic'/'Glenlea', RL4452/'AC Domain' and Kenyon/86ISMN 2137, were extracted from lyophilized fresh leaf tissue using the DNeasy Plant DNA extraction kit (Qiagen, Toronto, Canada). For the BW278/'AC Foremost' population, DNA from lyophilized fresh leaf tissue was extracted using a modified ammonium acetate method as described previously [37]. Stock DNA concentration was estimated with a fluorometer using Hoechst 33258 stain and diluted to a working concentration of $15 \mathrm{ng} / \mu \mathrm{l}$. All of the SNPs identified were genotyped using the KASP assay [38]. Using DNA sequence flanking the variant SNP position, two allele-specific forward primers and one common reverse primer were designed (Additional file 1: Table S1). PCR conditions and KASP assays were performed using methods as previously described [37]. Fluorescence detection of the PCR products was performed using an Omega Fluorostar plate reader (BMG LABTECH GmbH, Ortenberg, Germany). The data were analyzed using KlusterCaller software (LGC Genomics, Beverly, USA). Only SNP markers that showed high quality allele calls were used for linkage analysis. The quality of the marker was determined based on the cluster quality of the scatter plot and by comparing the allele call of each genotype with alleles of the parental lines. Datapoints that did not fit within clusters were scored as missing data and were excluded from linkage analysis. 
PCR conditions and genotyping methods for SSR markers were previously described [14], and the same protocol was used to test the sequence characterized amplified region (SCAR) marker pwm6 and the ConservedPrimer marker pwm16.

\section{Linkage analysis}

A linkage map of the region of chromosome arm 2BS carrying Lr16 was constructed for each mapping population using MapDisto 1.7.7 software [39]. Loci were analyzed for conformation to a Mendelian segregation ratio (1:1) using a $X^{2}$ test. A minimum LOD (logarithm of odds) threshold of 3.0 and maximum recombination fraction of 0.3 were used to identify linkage groups. Recombination fractions were converted into map distances using the Kosambi mapping function [40].

\section{Haplotype analysis of wheat lines with Lr16 SNP markers} Marker haplotype analysis was performed on a panel of 133 wheat lines and cultivars to evaluate the diagnostic potential of SNP markers linked with Lr16 for MAS. This collection of wheat germplasm was assembled by Dr. D.J. Somers, formerly of Agriculture and Agri-Food Canada, from western Canadian wheat breeders. The wheat cultivars and breeding lines were previously tested with $P$. triticina isolate 12-3 MBDS and have known infection types. The panel consists of cultivars and breeding lines from nine Canadian wheat marketing classes, which represent different grain quality profiles for different end-uses. Considerable diversity exists between these marketing classes. The haplotype panel also included wheats from 10 additional countries to further broaden the diversity sampled (Table 2 and Additional file 1: Table S2). Most wheat lines in the haplotype panel were susceptible to isolate 12-3 MBDS (i.e., do not carry Lr16). The few resistant lines included in the haplotype panel were known carriers of $\operatorname{Lr} 16$.

\section{Results}

\section{Phenotypic evaluation}

Each of the mapping populations (BW278/'AC Foremost' RIL, 'Majestic'/'Glenlea' DH, RL4452/'AC Domain' DH, and 'Kenyon'/86ISMN2137 RIL) fitted a 1 resistant : 1 susceptible segregation ratio, indicating single gene segregation for resistance to $P$. triticina isolate 12-3 MBDS (Table 1). Although each of these parental lines carries a number of leaf rust resistance genes, the isolate used here (12-3 MBDS) was virulent on those genes, except for Lr16 on which it was avirulent. Resistant lines in these populations had the infection type ' 1 ' with a large necrotic ring around a small uredinium, characteristic of Lr16, whereas susceptible lines had infection types ' 3 ' or ' 4 '.
Table 1 Segregation of Lr16 in four wheat populations, including $X^{2}$ for fit to a 1:1 ratio and corresponding probability

\begin{tabular}{llllll}
\hline Population & Resistant & Susceptible & Total & $X_{1: 1}^{2}$ & $P$ \\
\hline BW278/'AC Foremost' & 187 & 197 & 384 & 0.260 & 0.610 \\
'AC Majestic'/'Glenlea' & 196 & 204 & 400 & 0.160 & 0.689 \\
RL4452/'AC Domain' & 91 & 80 & 171 & 0.708 & 0.400 \\
'Kenyon'/86ISMN 2137 & 42 & 45 & 87 & 0.103 & 0.748 \\
\hline
\end{tabular}

\section{Marker development}

The SCAR marker pwm6 was developed from the DArT marker wPt-5960 that mapped near Lr16, and the ConservedPrimer marker pwm16 was developed from the wEST BF483211 that was mapped on the distal end of chromosome 2BS.

SNP markers were identified from publicly available wheat genomic resources and SNP databases using sequences of previously mapped markers linked to the Lr16 locus on chromosome 2BS, syntenic genes of Brachypodium and rice genomes and from RGAs identified from RNA-seq and in silico subtraction. SNPs were also identified from sequences of RGAs that were tightly linked with Lr16. A total of 219 putative SNP loci were identified. Of these, 83 SNPs were mined from CerealsDB using wESTs, SSRs, and DArTs located on chromosome arm 2BS as BLAST queries or were reported on CerealsDB as tentatively mapped to chromosome arm 2BS [29], 35 SNPs from $90 \mathrm{~K}$ iSelect Infinium Array [28], six SNPs were from RGAs identified through RNA-seq and in silico subtraction analysis [36], 64 SNPs were from the exome sequence of BW278/'AC Foremost' population [32], and 21 SNPs were identified from CerealsDB or the $90 \mathrm{~K}$ wheat Infinium SNP array using syntenic genes in Brachypodium and rice genomes as BLAST queries. Additionally, 10 SNP markers were derived from coding sequence (CDSs) of RGAs tightly linked with Lr16. KASP assays were designed for SNPs and tested on the parents of the four mapping populations, which yielded $70(32 \%)$ polymorphic SNPs that were tested for linkage.

\section{Lr16 genetic linkage map}

A total of 43 high quality new SNP markers and two PCR markers (SCAR pwm6 and ConservedPrimer marker pwm16) were mapped on chromosome arm 2BS linked with the resistance gene $\operatorname{Lr} 16$ across the four mapping populations (Fig. 1; Additional file 1: Table S2). The SNPs were selected based on cluster quality of the allele calls as revealed on the KASP genotyping assay. Three SSR markers (wmc764, gwm210 and wmc661) were previously mapped relative to Lr16 [14]. Genetic positions and marker order were consistent across the four populations (Fig. 1) and no marker deviated from the expected 1:1 Mendelian segregation ratio (data not 


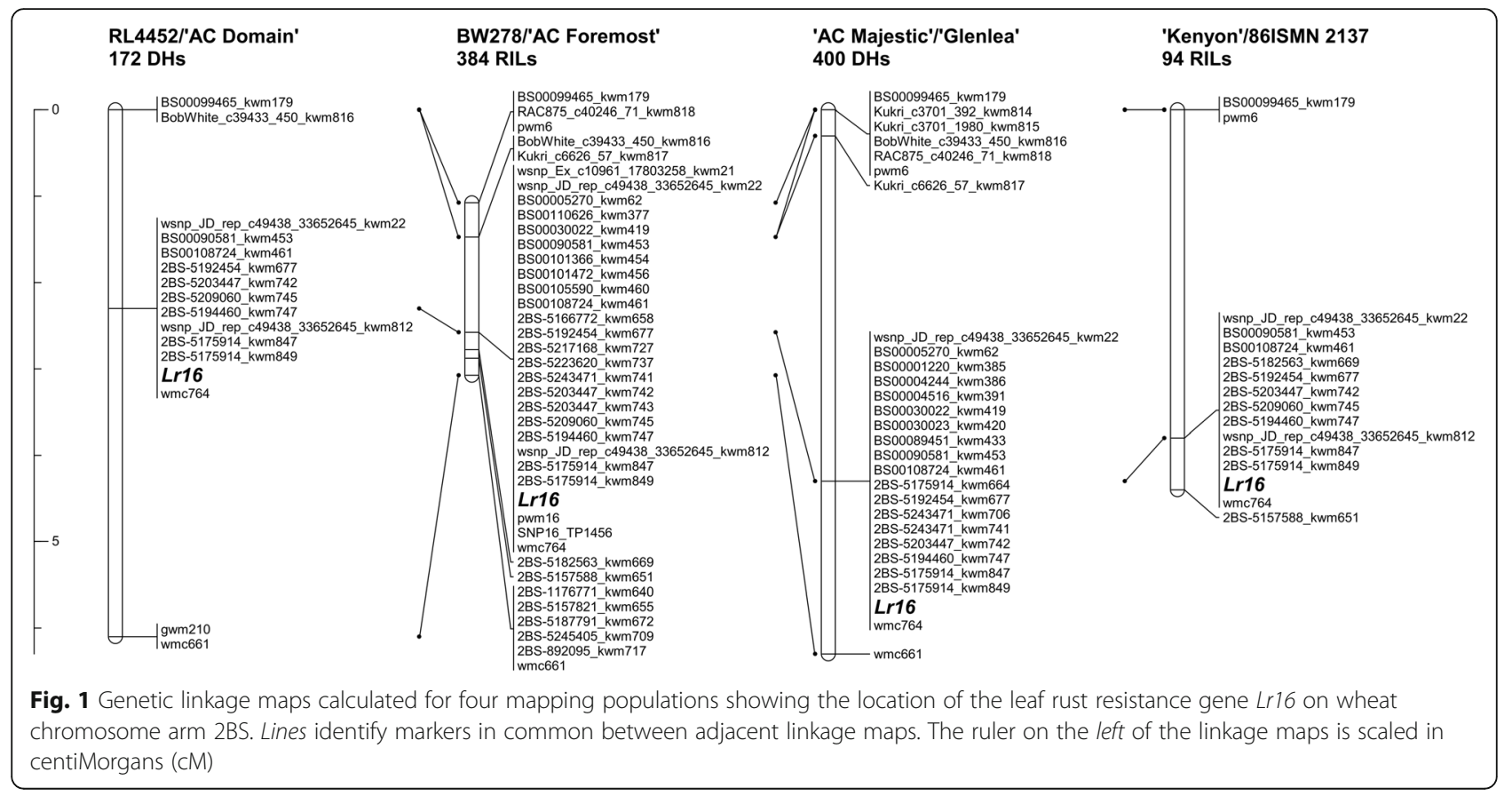

shown). In all four populations, $\operatorname{Lr} 16$ was flanked distally by the SNP marker BS00099465_kwm179 while the SSR marker wmc661 was the proximal flanking marker in all populations except 'Kenyon'/86ISMN 2137. The SNP marker 2BS-5157588_kwm651 flanked Lr16 proximally in both the 'Kenyon'/86ISMN 2137 and BW278/'AC Foremost' populations. It is worth noting that 2BS5182563_kwm669 was the closest proximal flanking marker of Lr16 in BW278/'AC Foremost' while it cosegregated with $\operatorname{Lr} 16$ in 'Kenyon'/86ISMN 2137. This result was not unexpected given the higher genetic resolution of BW278/'AC Foremost', which consisted of 384 RILs (i.e., approximately equivalent to 768 gametes). Similarly, the SNP marker Kukri_c6626_57_kwm817 was the closest distal flanking marker in the BW278/'AC Foremost' and 'AC Majestic'/'Glenlea' populations. One SSR and nine SNP markers were mapped in all the four populations while the remaining markers were mapped in one, two or three populations (Fig. 1). Of the ten markers mapped in all populations, an SSR (wmc764) and eight SNP markers (wsnp_JD_rep_c49438_33652645_kwm22, BS00090581_kwm453, BS00108724_kwm461, 2BS-51924 54_kwm677, 2BS-5203447_kwm742, 2BS-5194460_kwm 747, 2BS-5175914_kwm847, and 2BS-5175914_kwm849) co-segregated with Lr16. Five of the co-segregating SNPs were derived from three RGAs found in the Lr16 region. The SNPs BS00108724_kwm461, 2BS-5175914_kwm847, and 2BS-5175914_kwm849 were derived from the same RGA, while 2BS-5203447_kwm742 and 2BS-5194460_ kwm747 were from two additional RGAs. A SNP marker identified from RNA-seq and in silico subtraction analysis
(SNP16_TP1456) co-segregated with Lr16 in the BW278/ 'AC Foremost' population. SNP16_TP1456 and 2BS5194460_kwm747 were derived from the same RGA.

\section{Haplotype analysis}

The eight SNP markers that co-segregated with $\operatorname{Lr} 16$ in all four populations were tested on a diverse set of 133 wheat lines and cultivars (Additional file 1: Table S2). All lines and cultivars were previously tested with leaf rust race 12-3 MBDS and have a known genotype at the Lr16 locus. The panel was comprised of 10 resistant lines $(\operatorname{Lr} 16+)$, while the remaining lines were susceptible (Lr16-) (Table 2). Six of the SNP markers (BS00108724 kwm461, 2BS-5192454_kwm677, 2BS-5203447_kwm742, 2BS-5194460_kwm747, 2BS-5175914_kwm847, and 2B S-5175914_kwm849) were consistent with Lr16 phenotype and were completely predictive of $L r 16$ for all wheat lines/cultivars in the panel (Table 2). All these markers have exhibited high quality clustering as KASP assays when testing homozygous lines (Fig. 2). Of the six predictive SNP markers, 2BS-5194460_kwm747, 2BS5192454_kwm677, 2BS-5175914_kwm847, and 2BS5175914_kwm849 were best for distinguishing heterozygotes (Additional file 2: Figure S1) and are well suited for MAS of Lr16 in wheat breeding programs.

\section{Discussion}

In the present work, we were able to develop numerous new markers that delineate the Lr16 interval on the distal end of the short arm of chromosome 2B. A higher resolution SNP linkage map for Lr16 was developed 


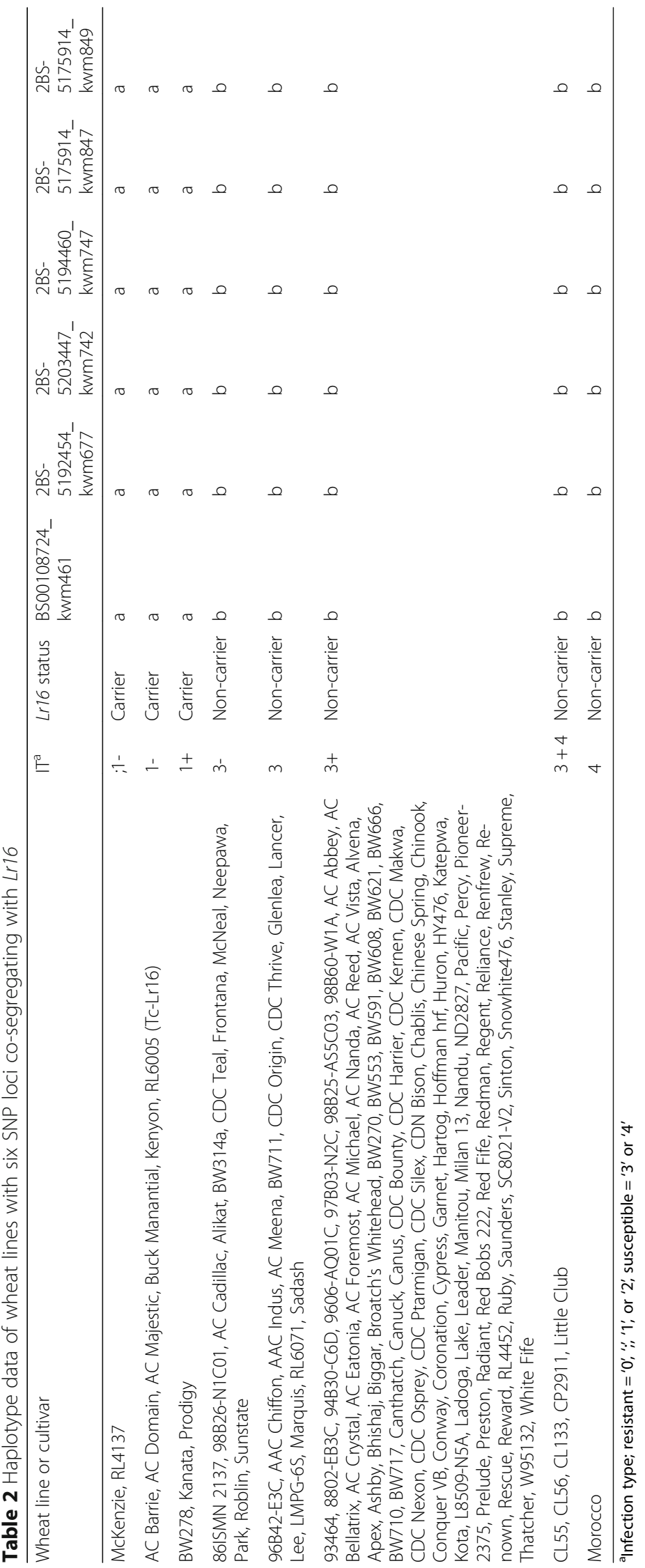




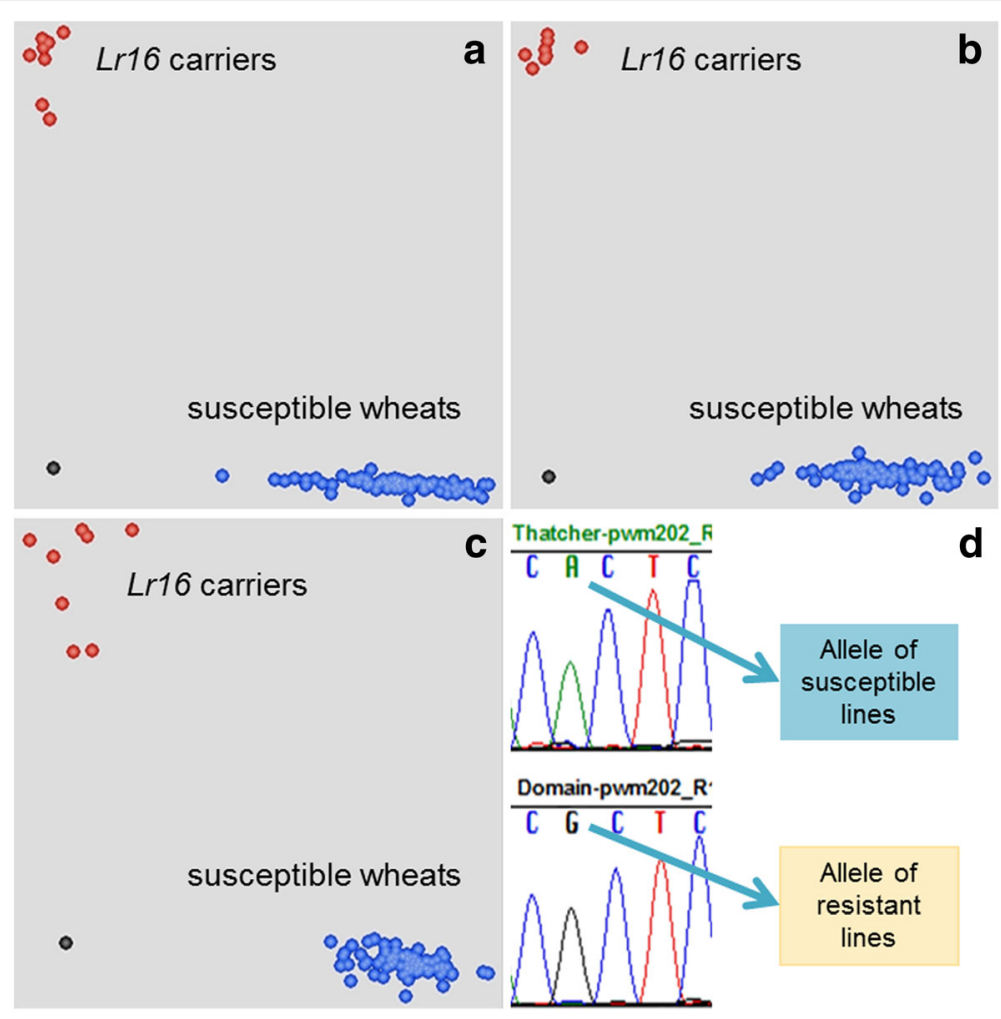

Fig. 2 Cartesian cluster plots of KASP markers (a) 2BS-5175914_kwm849, (b) 2BS-5192454_kwm677, (c) 2BS-5175914_kwm847 tested on a set of 133 wheat lines to show the diagnostic potential of SNP markers for high-throughput MAS. The $x$-axis and $y$-axis are the fluorescence intensities of FAM and HEX, respectively. Black datapoints are no template controls. d Sanger sequencing chromatograms, for 'Thatcher' and 'AC Domain', of an RGA co-segregating with Lr16 from which the SNP marker 2BS-5175914_kwm849 was identified

using four independent mapping populations, which represent approximately 768 (BW278/'AC Foremost', 384 RILs), approximately 188 ('Kenyon'/86ISMN 2137, 94 RILs), 400 ('AC Majestic'/'Glenlea', $400 \mathrm{DHs)}$ and 172 (RL4452/'AC Domain', 172 DHs) gametes (Fig. 1). In total, 43 SNP and two PCR markers were mapped in the Lr16 interval that was localized within $\sim 1.8 \mathrm{cM}$.

Lr16 is a race-specific seedling stage resistance gene [14]. Previously characterized seedling stage leaf rust resistance genes (e.g., Lr1, Lr10 and Lr21) were found to be RGAs [9-11]. The majority of wheat $R$ genes have coiled coil (CC), nucleotide-binding-site (NBS), and leucine-rich-repeat (LRR) motifs, and are referred to as CNL type $\mathrm{R}$ genes. Various studies have reported that $\mathrm{R}$ genes are often present in clusters in the genomes of many species. Here, we identified three RGA-like genes clustered near the Lr16 locus and five SNP markers (BS00108724_kwm461, 2BS-5203447_kwm742, 2BS5194460_kwm747, 2BS-5175914_kwm847, and 2BS5175914_kwm849), which were developed from these RGAs and co-segregated with Lr16 in all mapping populations (Fig. 1).

Stacking (or pyramiding) of multiple resistance genes is necessary to prolong resistance durability and enhance effective use of $L r$ genes [2]. $L r 16$ has been shown to confer effective resistance in leaf rust nurseries when combined with adult-plant $\mathrm{R}$ genes such as Lr34 and Lr13 [16, 18, 41]. In fact, Lr16 does not provide effective resistance in Canada when used as the sole source of leaf rust resistance [42]. Thus, diagnostic molecular markers are necessary to stack $L r 16$ with other $L r$ genes through MAS. Here we found six SNP markers that were predictive of the presence or absence of Lr16 in the diverse set of wheat germplasm tested (Table 2 and Additional file 1: Table S2). KASP assays were designed for SNPs that can readily be used in wheat breeding programs. The utility of the SNP markers was confirmed using a panel of 133 wheat lines/cultivars. The panel included reference stocks, spelt wheat, and a diverse set of cultivars and breeding lines from Canada, USA, Asia, Australia, North Africa, and South America with known genotype at the Lr16 locus (Additional file 1: Table S2). The Canadian wheat cultivars and breeding lines comprised different marketing classes with different end-use functionality.

Lr16 is believed to be originally sourced from five wheat cultivars, 'Columbus', 'Warden', 'Exchange, 'Selkirk', and 'Etoile de Choisy' [36]. 'AC Domain', 'Kanata' and 'Columbus' inherited Lr16 from RL4137. Subsequently, 
the wheat line BW278 inherited Lr16 from 'AC Domain', while 'AC Barrie,' 'AC Majestic', 'McKenzie' and 'Prodigy' inherited Lr16 from 'Columbus'. For Kenyon, the source of Lr16 was 'Buck Manantial'. The wheat cultivar 'Buck Manantial' was originally released in Argentina and its source of Lr16 could not be determined by the authors. The Thatcher-Lr16 differential line RL6005 inherited Lr16 from 'Exchange'. Interestingly, both Kenyon and RL6005 have a different allele for the SSR marker wmc764 than the other Lr16 carriers in this study [14]. The polymorphism at wmc764 between the $\operatorname{Lr} 16$ carriers is most likely a relatively recent mutation since SNPs in the $\operatorname{Lr} 16$ region do not differentiate the different sources of $\operatorname{Lr} 16$. Six SNP markers (BS00108724_kwm461, 2BS-5192454_ kwm677, 2BS-5203447_kwm742, 2BS-5194460_kwm747, 2BS-5175914_kwm847, and 2BS-5175914_kwm849) presented in this work were completely predictive of Lr16 in a diverse set of wheat germplasm and will be useful for MAS of Lr16. Interestingly, all the markers except 2BS5192454_kwm677 were derived from RGAs (Additional file 1: Table S1). These new SNP markers will be useful in fine mapping and cloning of $\operatorname{Lr} 16$.

\section{Conclusions}

Leaf rust resistance is a high priority for wheat breeders across the world. Lr16 is a widely deployed R gene that provides effective resistance when pyramided with Lr34. Lr16 was mapped to chromosome arm $2 \mathrm{BS}$ relative to numerous SNP markers in four mapping populations. The position of Lr16 relative to the DNA markers was consistent in all crosses. Six SNPs co-segregating with Lr16 were identified that are individually predictive for the presence/absence of Lr16 in a diverse set of 133 wheat lines. In this study, several approaches were used to develop markers, including (1) using previously mapped SSR, DArT and SNP markers, (2) developing conserved primers using wEST, (3) mining SNP markers based on sequences of previously mapped markers or comparative synteny analysis with Brachypodium and rice, and (4) RGA-based SNP marker development. Of the 229 SNPs identified in this study, only 16 SNPs (7\%) were developed from RGAs. However, five of the six predictive and co-segregating SNP markers were derived from RGAs. This demonstrates that RGA-based marker development is an effective approach for fine mapping and further cloning of resistance genes. The new predictive SNP markers developed here will enable efficient selection of $L r 16$ in wheat breeding programs and will be useful for further cloning efforts of $\operatorname{Lr} 16$.

\section{Additional files}

Additional file 1: Table S1. PCR primers for DNA markers used in the study. Table S2. Lr16 haplotype panel sorted by Lr16 status, country of origin, marketing class, and cultivar/line name. (XLSX $39 \mathrm{~kb}$ )
Additional file 2: Figure S1. Genotyping profile of the KASP markers (A) 2BS-5194460_kwm747, (B) 2BS-5192454_kwm677, (C) 2BS5175914_kwm847, (D) 2BS-5175914_kwm849, (E) 2BS-5203447_kwm742, and (F) BS00108724_kwm461 tested on homozygous Lr16 carriers, homozygous susceptible wheats, and heterozygous plants to show the diagnostic potential of the KASP assays to distinguish between heterozygous and homozygous samples. (PPTX $826 \mathrm{~kb}$ )

\section{Abbreviations}

BSA: Bulked segregant analysis; CC-NB-LRR: Coiled-coil, nucleotide binding site, leucine rich repeat; $\mathrm{CNL}$ : Coiled-coil, nucleotide binding site, leucine rich repeat; CWRS: Canada western red spring; DArT: Diversity arrays technology; DH: Doubled haploid; KASP: Kompetitive allele specific PCR; MAS: Markerassisted selection; R: Resistance; RGA: Resistance gene analog;

RIL: Recombinant inbred line; SNP: Single nucleotide polymorphism; SSR: Simple sequence repeat; WEC: Whole exome capture; WEST: Wheat expressed sequence tag

\section{Acknowledgements}

The authors thank technical staff from the participating labs for their contributions to this research. Based upon research contributions from the National Research Council Canada, this publication was given the contribution number NRCC \#56266.

\section{Funding}

This research was funded by AAFC Agriflex project 2521, AAFC Genomics R\&D Initiative (GRDI) project J-000006, the Canadian Wheat Improvement Flagship (NRC's contribution to the Canadian Wheat Alliance), and as part of CTAG and $C T A G^{2}$, Genome Prairie projects funded by Genome Canada, Manitoba Agriculture, Food and Rural Development, Manitoba Wheat and Barley Growers Association, Saskatchewan Ministry of Agriculture, and Western Grain Research Foundation. These funding bodies did not participate in the design of the study and collection, analysis, and interpretation of data, or in writing the manuscript.

\section{Availability of data and materials}

All supporting data are included as an additional file. The datasets during and/or analyzed during the current study are available from the corresponding author upon reasonable request.

\section{Authors' contributions}

CAM, BDM, CWH, JPF, MTK, FMY, CJP, JGM, DGH, PRF, and AGS planned and organized the study. MTK, CAM, FMY, CJP, NRH, and JPF designed DNA markers and collected marker data. BDM collected leaf rust data. JGM, DGH, and CAM developed mapping populations. CAM and MTK conducted linkage analysis. All authors contributed to and approved the final manuscript.

\section{Competing interests}

The authors declare that they have no competing interests.

\section{Consent for publication}

Not applicable.

Ethics approval and consent to participate

Not applicable.

\footnotetext{
Author details

'Agriculture and Agri-Food Canada, Morden Research and Development Centre, 101 Route 100, Morden, MB R6M 1Y5, Canada. ${ }^{2}$ National Research Council, 110 Gymnasium Place, Saskatoon, SK S7N 0W9, Canada. ${ }^{3}$ University of Saskatchewan, Crop Development Centre, 51 Campus Drive, Saskatoon, SK S7N 5A8, Canada. ${ }^{4}$ University of Saskatchewan, Global Institute for Food Security, 110 Gymnasium Place, Saskatoon, SK S7N 4J8, Canada. ${ }^{5}$ Agriculture and Agri-Food Canada, Ottawa Research and Development Centre, 1341 Baseline Road, Ottawa, ON K1A 0C5, Canada. ${ }^{6}$ Department of Plant Pathology, Kansas State University, Manhattan, KS 66506, USA. 'USDA-ARS, Hard Winter Wheat Genetics Research Unit, Manhattan, KS 66506, USA.
} 
Received: 27 September 2016 Accepted: 31 January 2017 Published online: 15 February 2017

\section{References}

1. CGIAR_Research_Program_on_Wheat. Wheat CRP Annual Report 2013: the vital grain of civilization and food security. Mexico: Consultative Group on International Agricultural Research; 2014.

2. Ellis JG, Lagudah ES, Spielmeyer W, Dodds PN. The past, present and future of breeding rust resistant wheat. Front Plant Sci. 2014:5:641.

3. Bolton MD, Kolmer JA, Garvin DF. Wheat leaf rust caused by Puccinia triticina. Mol Plant Pathol. 2008;9(5):563-75.

4. Samborski DJ. Wheat leaf rust. In: Roelfs AP, Bushnell WR, editors. The cereal rusts, vol. 2. New York: Academic; 1985. p. 39-59.

5. Oliver RP. A reassessment of the risk of rust fungi developing resistance to fungicides. Pest Manag Sci. 2014;70(11):1641-5.

6. Herrera-Foessel SA, Huerta-Espino J, Calvo-Salazar V, Lan CX, Singh RP. Lr72 confers resistance to leaf rust in durum wheat cultivar Atil C2000. Plant Dis. 2014;98(5):631-5.

7. Park RF, Mohler V, Nazari K, Singh D. Characterisation and mapping of gene Lr73 conferring seedling resistance to Puccinia triticina in common wheat. Theor Appl Genet. 2014;127(9):2041-9.

8. Singh D, Mohler V, Park RF. Discovery, characterisation and mapping of wheat leaf rust resistance gene Lr71. Euphytica. 2012;190(1):131-6.

9. Cloutier S, McCallum BD, Loutre C, Banks TW, Wicker T, Feuillet C, Keller B, Jordan MC. Leaf rust resistance gene $L r 1$, isolated from bread wheat (Triticum aestivum L.) is a member of the large psr567 gene family. Plant Mol Biol. 2007;65(1-2):93-106.

10. Feuillet C, Travella S, Stein N, Albar L, Nublat A, Keller B. Map-based isolation of the leaf rust disease resistance gene $L$ r10 from the hexaploid wheat (Triticum aestivum L.) genome. Proc Natl Acad Sci U S A. 2003;100(25): 15253-8.

11. Huang L, Brooks SA, Li W, Fellers JP, Trick HN, Gill BS. Map-based cloning of leaf rust resistance gene $L \mathrm{r} 21$ from the large and polyploid genome of bread wheat. Genetics. 2003;164(2):655-64.

12. Krattinger SG, Lagudah ES, Spielmeyer W, Singh RP, Huerta-Espino J, McFadden $H$, Bossolini E, Selter LL, Keller B. A putative ABC transporter confers durable resistance to multiple fungal pathogens in wheat. Science. 2009:323(5919):1360-3.

13. Moore JW, Herrera-Foessel S, Lan C, Schnippenkoetter W, Ayliffe M, HuertaEspino J, Lillemo M, Viccars L, Milne R, Periyannan S, et al. A recently evolved hexose transporter variant confers resistance to multiple pathogens in wheat. Nat Genet. 2015;47(12):1494-8.

14. McCartney CA, Somers DJ, McCallum BD, Thomas J, Humphreys DG, Menzies JG, Brown PD. Microsatellite tagging of the leaf rust resistance gene Lr16 on wheat chromosome 2BS. Mol Breed. 2005;15(4):329-37.

15. McCallum BD, Seto-Goh P. Physiological specialization of wheat leaf rust [Puccinia triticina] in Canada in 2000. Can J Plant Pathol. 2003;25:91-7.

16. German SE, Kolmer JA. Effect of gene Lr34 in the enhancement of resistance to leaf rust of wheat. Theor Appl Genet. 1992;84(1-2):97-105.

17. Hiebert CW, Thomas JB, McCallum BD. Stacking pairs of disease resistance genes in wheat populations using telocentric chromosomes. Mol Breed. 2010;26(4):681-92.

18. Kolmer J. Enhanced leaf rust resistance in wheat conditioned by resistance gene pairs with Lr13. Euphytica. 1992;61:123-30.

19. Kolmer JA, Liu JQ. Inheritance of leaf rust resistance in the wheat cultivars AC Majestic, AC Splendor, and AC Karma. Can J Plant Pathol. 2002;24:327-31.

20. Somers DJ, Isaac P, Edwards K. A high-density microsatellite consensus map for breeding wheat (Triticum aestivum L.). Theor Appl Genet. 2004;109:1105-14.

21. Thomas JB, DePauw RM, Knox RE, Czarnecki E, Campbell AB, Nielsen J, McKenzie RIH, Degenhardt KJ, Morrison RJ. AC foremost red spring wheat. Can J Plant Sci. 1997;77(4):657-60

22. Evans LE, Shebeski LH, McGinnis RC, Briggs KG, Zuzens D. Glenlea red spring wheat. Can J Plant Sci. 1972:52:1081-2.

23. Townley-Smith TF, Czarnecki EM. AC Domain hard red spring wheat. Can Plant Sci. 2008:88(2):347-50.

24. Hughes GR, Hucl P. Kenyon hard red spring wheat. Can J Plant Sci. 1991; 71(4):1165-8.

25. McCallum BD, Seto-Goh P, Xue A. Physiologic specialization of Puccinia triticina, the causal agent of wheat leaf rust, in Canada in 2009. Can J Plant Pathol. 2013;35(3):338-45.
26. Long DL, Kolmer JA. A North American system of nomenclature for Puccinia recondita f. sp. tritici. Phytopathology. 1989:79(5):525-9.

27. You FM, Huo N, Gu YQ, Lazo GR, Dvorak J, Anderson OD. ConservedPrimers 2.0: a high-throughput pipeline for comparative genome referenced intronflanking PCR primer design and its application in wheat SNP discovery. BMC Bioinf. 2009;10:331.

28. Wang S, Wong D, Forrest K, Allen A, Chao S, Huang BE, Maccaferri M, Salvi S, Milner SG, Cattivelli L, et al. Characterization of polyploid wheat genomic diversity using a high-density 90,000 single nucleotide polymorphism array. Plant Biotechnol J. 2014;12(6):787-96.

29. Wilkinson PA, Winfield MO, Barker GL, Allen AM, Burridge A, Coghill JA, Edwards KJ. CerealsDB 2.0: an integrated resource for plant breeders and scientists. BMC Bioinf. 2012;13:219.

30. National Center for Biotechnology Information. http://www.ncbi.nlm.nih.gov (2016). Accessed 12 Aug 2016.

31. GrainGenes: A Database for Triticeae and Avena. https://wheat.pw.usda.gov/ GG3/ (2016). Accessed 12 Aug 2016.

32. Jordan KW, Wang S, Lun Y, Gardiner LJ, MacLachlan R, Hucl P, Wiebe K, Wong D, Forrest KL, Consortium I, et al. A haplotype map of allohexaploid wheat reveals distinct patterns of selection on homoeologous genomes. Genome Biol. 2015;16:48.

33. Consortium TIWGS. A chromosome-based draft sequence of the hexaploid bread wheat (Triticum aestivum) genome. Science. 2014;345(6194). doi: 10. 1126/science.1251788.

34. Besemer J, Borodovsky M. GeneMark: web software for gene finding in prokaryotes, eukaryotes and viruses. Nucleic Acids Res. 2005;33 suppl 2: W451-4.

35. Li P, Quan X, Jia G, Xiao J, Cloutier S, You FM. RGAugury: A pipeline for genome-wide prediction of resistance gene analogs (RGAs) in plants. BMC Genomics. 2016;17:accepted.

36. Harrison NR, Fritz AK, Glasscock Jl, Ahmed S, Messina DN, Amand PS, Fellers JP. Using RNA sequencing and in silico subtraction to identify resistance gene analog markers for Lr16 in wheat. Plant Genome. 2015;8(2). doi: 10. 3835/plantgenome2014.08.0040.

37. Kassa MT, You FM, Fetch TG, Fobert P, Sharpe A, Pozniak CJ, Menzies JG, Jordan MC, Humphreys G, Zhu T, et al. Genetic mapping of SrCad and SNP marker development for marker-assisted selection of Ug99 stem rust resistance in wheat. Theor Appl Genet. 2016;129(7):1373-82.

38. Allen AM, Barker GLA, Berry ST, Coghill JA, Gwilliam R, Kirby S, Robinson P, Brenchley RC, D'Amore R, McKenzie N, et al. Transcript-specific, singlenucleotide polymorphism discovery and linkage analysis in hexaploid bread wheat (Triticum aestivum L.). Plant Biotechnol J. 2011;9(9):1086-99.

39. Lorieux M. MapDisto: fast and efficient computation of genetic linkage maps. Mol Breed. 2012;30(2):1231-5.

40. Kosambi DD. The estimation of map distances from recombination values. Ann Eugen. 1944;12(1):172-5.

41. Samborski DJ, Dyck PL. Enhancement of resistance to Puccinia recondita by interactions of resistance genes in wheat. Can J Plant Pathol. 1982:4(2):152-6.

42. McCallum BD, DePauw RM. A review of wheat cultivars grown in the Canadian prairies. Can J Plant Sci. 2008;88(4):649-77.

\section{Submit your next manuscript to BioMed Central and we will help you at every step:}

- We accept pre-submission inquiries

- Our selector tool helps you to find the most relevant journal

- We provide round the clock customer support

- Convenient online submission

- Thorough peer review

- Inclusion in PubMed and all major indexing services

- Maximum visibility for your research

Submit your manuscript at www.biomedcentral.com/submit 